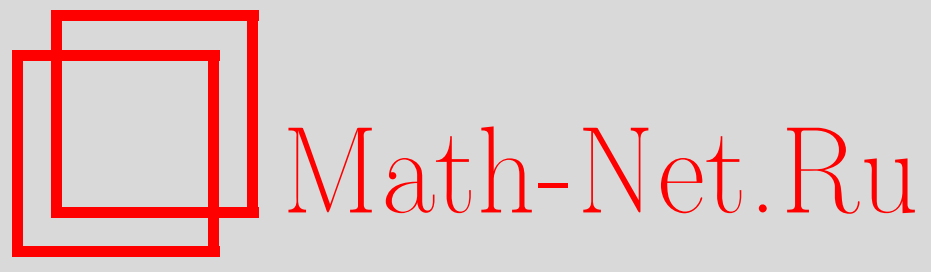

А. А. Васильева, Многозначные отображения со вторым модулем непрерывности, Матем. заметки, 2007, том 82, выпуск 5, 787-791

DOI: https://doi.org/10.4213/mzm4092

Использование Общероссийского математического портала Math-Net.Ru подразумевает, что вы прочитали и согласны с пользовательским соглашением http://www . mathnet.ru/rus/agreement

Параметры загрузки:

IP : 54.197 .130 .99

26 апреля 2023 г., 04:55:52

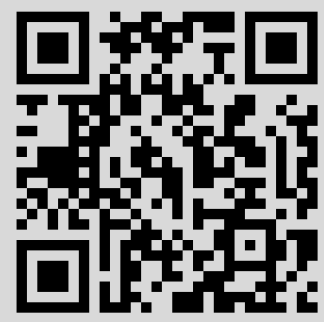




\section{Многозначные отображения со вторым модулем непрерывности}

\section{А. А. Васильева}

В этой работе изучаются свойства многозначных отображений со вторым модулем непрерывности и рассматривается вопрос существования липшицевой выборки. Достаточные условия существования липшицевой выборки рассматривались в [1]-[4] и других работах.

Пусть $(X, \rho)$ - метрическое пространство, $M_{1}, M_{2} \subset X$. Обозначим

$$
\begin{gathered}
e\left(M_{1}, M_{2}\right)=\sup _{x_{1} \in M_{1}} \inf _{x_{2} \in M_{2}} \rho\left(x_{1}, x_{2}\right), \quad h\left(M_{1}, M_{2}\right)=\max \left\{e\left(M_{1}, M_{2}\right), e\left(M_{2}, M_{1}\right)\right\} \\
\operatorname{dist}\left(M_{1}, M_{2}\right)=\inf _{x_{1} \in M_{1}, x_{2} \in M_{2}} \rho\left(x_{1}, x_{2}\right) .
\end{gathered}
$$

ОПРеДЕЛЕНИЕ 1 . Пусть $X_{1}, X_{2}$ - банаховы пространства, $A \subset X_{1}$ - выпуклое подмножество. Отображение $F: A \rightarrow 2^{X_{2}}$ удовлетворяет условию (1) (соответственно (2)), если оно имеет замкнутый график и существует возрастающая непрерывная функция $\nu: \mathbb{R}_{+} \rightarrow \mathbb{R}_{+}$такая, что $\int_{0}^{1} \nu(t) / t d t<\infty$ и для любых $x, y \in A$ выполнено

$$
e\left(F(x)+F(y), 2 F\left(\frac{x+y}{2}\right)\right) \leqslant\left\|\frac{x-y}{2}\right\|_{X_{1}} \nu\left(\left\|\frac{x-y}{2}\right\|_{X_{1}}\right)
$$

или соответственно

$$
h\left(F(x)+F(y), 2 F\left(\frac{x+y}{2}\right)\right) \leqslant\left\|\frac{x-y}{2}\right\|_{X_{1}} \nu\left(\left\|\frac{x-y}{2}\right\|_{X_{1}}\right) .
$$

ЗАмечАниЕ. Условие $\int_{0}^{1} \nu(t) / t d t<\infty$ эквивалентно тому, что $\sum_{n=1}^{\infty} \nu\left(l / 2^{n}\right)<\infty$ для любого $l>0$.

Если отображение $F$ удовлетворяет условию (1), то для любого $x \in A$ множество $F(x)$ выпукло и замкнуто. Если отображения $F$ и $G$ удовлетворяют условию (1) (или (2)), при этом $F+G$ имеет замкнутый график, то $F+G$ удовлетворяет (1) (соответственно (2)).

Пример 1. Пусть $M \subset X_{2}$ - выпуклое замкнутое множество, $c: A \rightarrow \mathbb{R}_{+}-$непрерывная функция такая, что для любых $x, y \in A$ выполнено

$$
\left|c(x)+c(y)-2 c\left(\frac{x+y}{2}\right)\right| \leqslant\left\|\frac{x-y}{2}\right\|_{X_{1}} \nu\left(\left\|\frac{x-y}{2}\right\|_{X_{1}}\right) .
$$

Тогда отображение $F(x)=c(x) M$ удовлетворяет условию (2).

ПримеР 2. Пусть отображение $F$ имеет выпуклый замкнутый график. Тогда оно удовлетворяет условию (1) с $\nu=0$.

Теорема 1. Пусть $F: A \rightarrow 2^{X_{2}}$ равномерно ограничено и удовлетворяет условию (1), $\widetilde{A} \subset A-$ выпуклое подмножество, $\operatorname{dist}(\widetilde{A}, \partial A)>0$. Тогда отображение $\left.F\right|_{\widetilde{A}}$ липшицево в хаусдорфовой метрике.

Если $A=[0,1], F(t)=[0, \sqrt{t}]$, то $F$ удовлетворяет условию $(1)$ с $\nu=0$, но не липшицево в хаусдорфовой метрике.

Работа выполнена при поддержке Российского фонда фундаментальных исследований (грант № 06-01-00160-a).

(C) А. А. ВАсильевА, 2007 
Теорема 2. Пусть $X$ - банахово пространство, $A \subset \mathbb{R}^{d}$ - выпуклый многогранник, $F: A \rightarrow 2^{X}$ - равномерно ограниченное отображсение, удовлетворяющее условию (2). Тогда оно липшичево в хаусдорфовой метрике. Если норма в $\mathbb{R}^{d}$ определяется как $\left\|\left(x_{1}, \ldots, x_{d}\right)\right\|=\sum_{k=1}^{d}\left|x_{k}\right|, A-\kappa у б$ со стороной длины $l$ и ограничение $F$ на множество вершин куба липшичево с константой $M$, то константа Липшица отображсения $F$ не превосходит $M+\sum_{n=1}^{\infty} \nu\left(l / 2^{n}\right)$.

Условие $\int_{0}^{1} \nu(t) / t d t<\infty$ существенно. Пусть $\nu_{0}(k h) \leqslant k \nu_{0}(h)$ для любых $h \geqslant 0, k \in \mathbb{N}$, и пусть

$$
\int_{0}^{1} \frac{\nu_{0}(t)}{t} d t=\infty, \quad \varphi(t)=\frac{t}{9} \int_{t}^{1} \frac{\nu_{0}(s)}{s} d s .
$$

Тогда отображение $F:[0,1] \rightarrow 2^{\mathbb{R}}, F(t)=\{\varphi(t)\}$, удовлетворяет (2) с $\nu=\nu_{0}$, но не является липшицевым (для $\nu_{0}=1$ это доказано в [5]).

Теорема 3. Пусть $K-d$-мерный куб, $F: K \rightarrow 2^{X}$ удовлетворяет условию (1). Тогда существует липшицева выборка из отображения $F$.

ДоказАтельство. Пусть $l$ - длина стороны куба. Построим выборку $f$ в вершинах куба произвольным образом. Выберем $M$ так, что для любых соседних вершин $x_{1}, x_{2}$ выполнено $\left\|f\left(x_{1}\right)-f\left(x_{2}\right)\right\| \leqslant M l$. Разделим каждое ребро пополам и разобьем $K$ на $2^{d}$ равных кубов $K_{j}$. Построим $f$ в их вершинах.

Пусть $\xi$ - одна из вершин куба $K_{j}, j=1, \ldots, 2^{d}$. Тогда найдется такое $0 \leqslant k_{\xi} \leqslant d$, что $\xi$ не принадлежит $\left(k_{\xi}-1\right)$-мерной грани и является центром $k_{\xi}$-мерной грани куба $K$. Если $k_{\xi}=0$, то $f(\xi)$ уже определена. Пусть $k_{\xi}=1$. Тогда $\xi=\left(x_{1}+x_{2}\right) / 2$, где $x_{1}, x_{2}-$ соседние вершины в $K$. Из условия (1) следует, что существует точка $y \in F(\xi)$ такая, что $\left\|f\left(x_{1}\right)+f\left(x_{2}\right)-2 y\right\| \leqslant l / 2 \nu(l / 2)$. Положим $f(\xi)=y$. Пусть $f$ построена в таких вершинах $\xi$, для которых $k_{\xi} \leqslant k$, при этом, если $\xi=\left(\xi_{1}+\xi_{2}\right) / 2$, где $\xi_{1}, \xi_{2}$ - центры параллельных $\left(k_{\xi}-1\right)$-мерных граней в $K$, то

$$
\left\|f\left(\xi_{1}\right)+f\left(\xi_{2}\right)-2 f(\xi)\right\| \leqslant c_{k_{\xi}} \frac{l}{2} \nu\left(\frac{l}{2}\right) .
$$

Построим $f$ в вершинах $\xi$, для которых $k_{\xi}=k+1$. Пусть $\xi$ является центром $(k+$ $1)$-мерной грани $\widetilde{K}$. Возьмем произвольные параллельные $k$-мерные грани $\widetilde{K}_{1}$ и $\widetilde{K}_{2}$, содержащиеся в $\widetilde{K}$, и обозначим их центры соответственно $x_{1}$ и $x_{2}$. Тогда $\xi=\left(x_{1}+x_{2}\right) / 2$. Из условия (1) следует, что существует такая точка $v \in F(\xi)$, что $\left\|f\left(x_{1}\right)+f\left(x_{2}\right)-2 v\right\| \leqslant$ $l / 2 \nu(l / 2)$. Положим $f(\xi)=v$. По построению для $\xi_{1}=x_{1}$ и $\xi_{2}=x_{2}$ выполнено $(3)$ с $c_{k_{\xi}}=$ 1. Пусть $\xi_{1}, \xi_{2}$ - центры двух других параллельных граней $\widetilde{K}_{3}$ и $\widetilde{K}_{4}$, содержащихся в $\widetilde{K}$. Проверим, что для них выполнено (3). В самом деле, проведем через точки $x_{1}$, $x_{2}, \xi_{1}$ и $\xi_{2}$ двумерную плоскость. Ее пересечение с кубом $K$ - это двумерный квадрат с вершинами $x, y, z$ и $w$, при этом $x_{1}=(x+y) / 2, x_{2}=(z+w) / 2, \xi_{1}=(y+z) / 2$, $\xi_{2}=(x+w) / 2$. Точки $x, y, z$ и $w$ являются центрами граней $\widetilde{K}_{1} \cap \widetilde{K}_{4}, \widetilde{K}_{1} \cap \widetilde{K}_{3}, \widetilde{K}_{2} \cap \widetilde{K}_{3}$ и $\widetilde{K}_{2} \cap \widetilde{K}_{4}$, поэтому в силу (3) выполнено

$$
\begin{array}{ll}
\left\|f(x)+f(y)-2 f\left(x_{1}\right)\right\| \leqslant c_{k} \frac{l}{2} \nu\left(\frac{l}{2}\right), & \left\|f(y)+f(z)-2 f\left(\xi_{1}\right)\right\| \leqslant c_{k} \frac{l}{2} \nu\left(\frac{l}{2}\right), \\
\left\|f(z)+f(w)-2 f\left(x_{2}\right)\right\| \leqslant c_{k} \frac{l}{2} \nu\left(\frac{l}{2}\right), & \left\|f(x)+f(w)-2 f\left(\xi_{2}\right)\right\| \leqslant c_{k} \frac{l}{2} \nu\left(\frac{l}{2}\right) .
\end{array}
$$

Таким образом,

$$
\begin{aligned}
& \left\|f\left(\xi_{1}\right)+f\left(\xi_{2}\right)-2 f(\xi)\right\| \\
& \quad \leqslant\left\|f\left(\xi_{1}\right)+f\left(\xi_{2}\right)-f\left(x_{1}\right)-f\left(x_{2}\right)\right\|+\frac{l}{2} \nu\left(\frac{l}{2}\right)
\end{aligned}
$$




$$
\begin{aligned}
\leqslant & \frac{1}{2}\|f(y)+f(z)+f(x)+f(w)-f(x)-f(y)-f(z)-f(w)\| \\
& +\frac{1}{2}\left\|f(y)+f(z)-2 f\left(\xi_{1}\right)\right\|+\frac{1}{2}\left\|f(x)+f(w)-2 f\left(\xi_{2}\right)\right\| \\
& +\frac{1}{2}\left\|f(x)+f(y)-2 f\left(x_{1}\right)\right\|+\frac{1}{2}\left\|f(z)+f(w)-2 f\left(x_{2}\right)\right\|+\frac{l}{2} \nu\left(\frac{l}{2}\right) \\
\stackrel{(4)}{\leqslant} 2 c_{k} & \frac{l}{2} \nu\left(\frac{l}{2}\right)+\frac{l}{2} \nu\left(\frac{l}{2}\right) .
\end{aligned}
$$

Значит, выполнено (3) с $c_{k+1}=2 c_{k}+1$. Так как $c_{1}=1$, то $c_{k}=2^{k}-1$.

Пусть $\xi, \eta$ - две произвольных соседних вершины в кубе $K_{j}$. Тогда одна из них является центром некоторой $k$-мерной грани $\widetilde{K}$, а другая - центром $(k-1)$-мерной грани, содержащейся в $\widetilde{K}$. Пусть $\xi$ - это центр $\widetilde{K}$, а $\eta$-центр грани $\widetilde{K}_{\eta} \subset \widetilde{K}$. Тогда $\xi=(\eta+\zeta) / 2$, где $\zeta$ - центр $(k-1)$-мерной грани $\widetilde{K}_{\zeta} \subset \widetilde{K}$, параллельной $\widetilde{K}_{\eta}$. Оценим $\|f(\xi)-f(\eta)\|$. Если $k=1$, то

$$
\begin{aligned}
\|f(\xi)-f(\eta)\| & \leqslant\left\|\frac{f(\eta)+f(\zeta)}{2}-f(\eta)\right\|+\left\|\frac{f(\eta)+f(\zeta)}{2}-f(\xi)\right\| \\
& \leqslant \frac{1}{2}\|f(\eta)-f(\zeta)\|+\frac{l}{4} \nu\left(\frac{l}{2}\right) \leqslant M \frac{l}{2}+\frac{l}{4} \nu\left(\frac{l}{2}\right) .
\end{aligned}
$$

Пусть для $k=k_{0}-1$ доказана оценка

$$
\|f(\xi)-f(\eta)\| \leqslant M \frac{l}{2}+\sum_{i=1}^{k-1} c_{i} \frac{l}{2} \nu\left(\frac{l}{2}\right)+\frac{1}{2} c_{k} \frac{l}{2} \nu\left(\frac{l}{2}\right) .
$$

Докажем, что аналогичная оценка выполнена для $k=k_{0}$. В самом деле, точка $\eta$ является серединой отрезка $\left[\eta_{1}, \eta_{2}\right]$, где $\eta_{i}$ - центры $(k-2)$-мерных параллельных граней $\widetilde{K}_{\eta, i} \subset \widetilde{K}_{\eta}$. Положим $\zeta_{i}=\eta_{i}+\zeta-\eta, \xi_{i}=\left(\eta_{i}+\zeta_{i}\right) / 2, \widetilde{K}_{\zeta, i}=\widetilde{K}_{\eta, i}+\zeta-\eta$. Пусть $\widetilde{K}_{i}-\left(k_{0}-1\right)$-мерная грань, образованная $\widetilde{K}_{\eta, i}$ и отрезком $\left[\eta_{i}, \zeta_{i}\right]$, где $i=1,2$. Тогда $\widetilde{K}_{\eta, i}$ и $\widetilde{K}_{\zeta, i}-$ параллельные $\left(k_{0}-2\right)$-мерные грани, содержащиеся в $\widetilde{K}_{i}$, и $\eta_{i}, \zeta_{i}$ являются их центрами. В силу (3)

$$
\left\|f\left(\eta_{1}\right)+f\left(\eta_{2}\right)-2 f(\eta)\right\| \leqslant c_{k_{0}-1} \frac{l}{2} \nu\left(\frac{l}{2}\right), \quad\left\|f\left(\zeta_{1}\right)+f\left(\zeta_{2}\right)-2 f(\zeta)\right\| \leqslant c_{k_{0}-1} \frac{l}{2} \nu\left(\frac{l}{2}\right) .
$$

По предположению индукции

$$
\begin{aligned}
& \left\|f\left(\xi_{i}\right)-f\left(\eta_{i}\right)\right\| \leqslant M \frac{l}{2}+\sum_{i=1}^{k_{0}-2} c_{i} \frac{l}{2} \nu\left(\frac{l}{2}\right)+\frac{1}{2} c_{k_{0}-1} \frac{l}{2} \nu\left(\frac{l}{2}\right), \\
& \left\|f\left(\xi_{i}\right)-f\left(\zeta_{i}\right)\right\| \leqslant M \frac{l}{2}+\sum_{i=1}^{k_{0}-2} c_{i} \frac{l}{2} \nu\left(\frac{l}{2}\right)+\frac{1}{2} c_{k_{0}-1} \frac{l}{2} \nu\left(\frac{l}{2}\right) .
\end{aligned}
$$

Значит,

$$
\begin{aligned}
\|f(\xi)-f(\eta)\| \leqslant & \frac{1}{2}\|f(\eta)-f(\zeta)\|+\frac{1}{2}\|f(\eta)+f(\zeta)-2 f(\xi)\| \\
\leqslant & \frac{1}{4}\left\|f\left(\eta_{1}\right)+f\left(\eta_{2}\right)-f\left(\zeta_{1}\right)-f\left(\zeta_{2}\right)\right\|+\frac{1}{4}\left\|f\left(\eta_{1}\right)+f\left(\eta_{2}\right)-2 f(\eta)\right\| \\
& \quad+\frac{1}{4}\left\|f\left(\zeta_{1}\right)+f\left(\zeta_{2}\right)-2 f(\zeta)\right\|+\frac{1}{2} c_{k_{0}} \frac{l}{2} \nu\left(\frac{l}{2}\right)
\end{aligned}
$$




$$
\begin{aligned}
& \stackrel{(5)}{\leqslant} \frac{1}{4}\left\|f\left(\eta_{1}\right)-f\left(\xi_{1}\right)\right\|+\frac{1}{4}\left\|f\left(\xi_{1}\right)-f\left(\zeta_{1}\right)\right\|+\frac{1}{4}\left\|f\left(\eta_{2}\right)-f\left(\xi_{2}\right)\right\| \\
&+\frac{1}{4}\left\|f\left(\xi_{2}\right)-f\left(\zeta_{2}\right)\right\|+\frac{1}{2} c_{k_{0}-1} \frac{l}{2} \nu\left(\frac{l}{2}\right)+\frac{1}{2} c_{k_{0}} \frac{l}{2} \nu\left(\frac{l}{2}\right) \\
& \stackrel{(6)}{\leqslant} M \frac{l}{2}+\sum_{i=1}^{k_{0}-2} c_{i} \frac{l}{2} \nu\left(\frac{l}{2}\right)+\frac{1}{2} c_{k_{0}-1} \frac{l}{2} \nu\left(\frac{l}{2}\right)+\frac{1}{2} c_{k_{0}-1} \frac{l}{2} \nu\left(\frac{l}{2}\right)+\frac{1}{2} c_{k_{0}} \frac{l}{2} \nu\left(\frac{l}{2}\right) \\
& \leqslant M \frac{l}{2}+\sum_{i=1}^{k_{0}-1} c_{i} \frac{l}{2} \nu\left(\frac{l}{2}\right)+\frac{1}{2} c_{k_{0}} \frac{l}{2} \nu\left(\frac{l}{2}\right) .
\end{aligned}
$$

Таким образом, если $\xi$ и $\eta$ - соседние вершины куба $K_{j}$, то

$$
\|f(\xi)-f(\eta)\| \leqslant M_{1} \frac{l}{2}, \quad \text { где } \quad M_{1}=M+\sum_{i=1}^{d-1} c_{i} \nu\left(\frac{l}{2}\right)+\frac{1}{2} c_{d} \nu\left(\frac{l}{2}\right) \leqslant M+2^{d+1} \nu\left(\frac{l}{2}\right)
$$

(так как $c_{k}=2^{k}-1,1 \leqslant k \leqslant d$ ). Пусть $K$ разбит на $2^{(n-1) d}$ равных кубов $K_{j}^{n-1}$, в вершинах которых построена выборка $f$, при этом если $\xi, \eta$ - соседние вершины, то

$$
|f(\xi)-f(\eta)| \leqslant M_{n-1} \frac{l}{2^{n-1}}, \quad \text { где } \quad M_{n-1} \leqslant M+2^{d+1} \sum_{k=1}^{n-1} \nu\left(\frac{l}{2^{k}}\right) .
$$

Каждый куб $K_{j}^{n-1}$ разбиваем на $2^{d}$ равных кубов $K_{j}^{n}$ и определяем в их вершинах функцию $f_{K_{j}^{n}}$. При этом из построения следует, что $f_{K_{j}^{n}}$ можно выбрать так, что если $K_{j}^{n}$ и $K_{i}^{n}$ пересекаются по некоторой грани $\widetilde{K}$, то $\left.f_{K_{j}^{n}}\right|_{\widetilde{K}}=\left.f_{K_{i}^{n}}\right|_{\widetilde{K}}$, так что функция $f(x):=f_{K_{j}^{n}}(x)$, $x \in K_{j}^{n}$, определена корректно. Если $\xi, \eta$ - соседние вершины $K_{j}^{n}$, то

$$
\|f(\xi)-f(\eta)\| \leqslant M_{n} \frac{l}{2^{n}}, \quad \text { где } \quad M_{n} \leqslant M_{n-1}+2^{d+1} \nu\left(\frac{l}{2^{n}}\right) \leqslant M+2^{d+1} \sum_{k=1}^{n} \nu\left(\frac{l}{2^{k}}\right) .
$$

Пусть $K$ имеет стороны, параллельные координатным осям, а в $\mathbb{R}^{d}$ задана норма $\|x\|_{1}=\sum_{i=1}^{d}\left|x_{i}\right|, x=\left(x_{1}, \ldots, x_{d}\right)$. Тогда $f$ липшицева с константой $M+2^{d+1} \sum_{n=1}^{\infty} \nu\left(l / 2^{n}\right)$. Продолжаем $f$ по непрерывности на весь куб $K$. Так как отображение $F$ имеет замкнутый график, то $f$ является выборкой из $F$. Так как все нормы в конечномерном пространстве эквивалентны, то $f$ будет липшицевой относительно любой другой нормы B $\mathbb{R}^{d}$.

Теорема 4. Пусть $A \subset \mathbb{R}^{d}$ - выпуклый компакт, $F: A \rightarrow 2^{X}$ - равномерно ограниченное отображение, удовлетворяющее условию (2). Пусть $A=\bigcup_{i=1}^{N} A_{i}$, где $A_{i}$ выпукльь, открыты в $A$ u

$$
L_{i}\left(\bar{A}_{i}\right)=\left\{\left(x_{1}, \ldots, x_{d}\right): 0 \leqslant x_{j} \leqslant 1,1 \leqslant j \leqslant d-1 ; 0 \leqslant x_{d} \leqslant f_{i}\left(x_{1}, \ldots, x_{d-1}\right)\right\},
$$

где $L_{i}$ - композиция гомотетии, поворота и сдвига в $\mathbb{R}^{d}, f_{i}:[0,1]^{d-1} \rightarrow \mathbb{R}$ - непрерьвная строго положительная функция такая, что выполнено

$$
\left|f_{i}(x)+f_{i}(y)-2 f_{i}\left(\frac{x+y}{2}\right)\right| \leqslant\|x-y\|_{\mathbb{R}^{d}} \nu\left(\|x-y\|_{\mathbb{R}^{d}}\right),
$$

где $\nu$ - такая же, как в определении условия (2). Тогда F липшичево в хаусдорфовой метрике и существует липшицева выборка из $F$. 


\section{СПИСОК ЦИТИРОВАННОЙ ЛИТЕРАТУРЫ}

[1] P. Shvartsman, Geom. Funct. Anal., 11:4 (2001), 840-868. [2] J.-P. Aubin, A. Cellina, Differential inclusions. Set-valued maps and viability theory, Grundlehren der Mathematischen Wissenschaften, 264, Springer-Verlag, Berlin, 1984. [3] J. Lindenstrauss, Michigan Math. J., 11:3 (1964), 263-287. [4] В. В. Чистяков, Матем. заметки, 71:4 (2002), 611-632. [5] В.К. Дзядык, Введение в теорию равномерного приближения функций полиномами, Наука, М., 1977.
А. А. Васильева
Московский государственный
университет им. М. В. Ломоносова
E-mail: vasilyeva_nastya@inbox.ru

Поступило

28.05.2007 